Pacific Journal of Mathematics

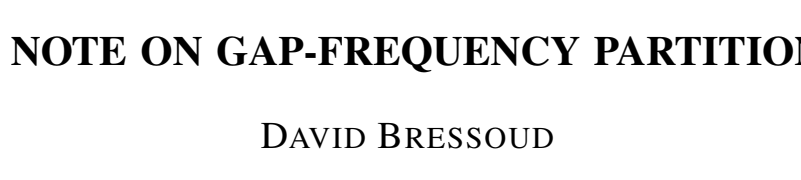




\title{
A NOTE ON GAP-FREQUENCY PARTITIONS
}

\author{
D. M. BRESSOUd
}

George Andrews has introduced gap-frequency partitions in order to interpret the Rogers-Selberg $q$-series identities related to the modulus seven. In this paper, we give a direct derivation of the generating function for such partitions. Our approach makes it much easier to extend and generalize the notion of gap-frequency partitions.

L. J. Rogers is known today primarily for his discovery of the Rogers-Ramanujan identities:

$$
\begin{aligned}
\prod_{\substack{n=1 \\
n \neq 0}}^{\infty}\left(1-q^{n}\right)^{-1} & =\sum_{m=0}^{\infty} \frac{q^{m^{2}}}{(q)_{m}}, \\
\prod_{\substack{n=1 \\
n \neq 0}}^{\infty}\left(1-q^{n}\right)^{-1} & =\sum_{m=0}^{\infty} \frac{q^{m^{2}+m}}{(q)_{m}},
\end{aligned}
$$

where $(a)_{\infty}=(a ; q)_{\infty}=\prod_{i=0}^{\infty}\left(1-a q^{i}\right)$,

$$
(a)_{m}=\frac{(a)_{\infty}}{\left(a q^{m}\right)_{\infty}}
$$

These analytic identities came to prominence largely because of $\mathrm{P}$. A. MacMahon's combinatorial interpretation of them:

(3) For $r=1$ or 2 , and any positive integer $n$, the partitions of $n$ into parts not congruent to $0, \pm r \bmod 5$ are equinumerous with the partitions of $n$ into parts with difference at least two between parts, and in which one appears as a part at most $r-1$ times.

Statement (3) can be proved from equations (1) and (2) by viewing each side of the equations as a generating function (see [3], §19.13).

It is less well known that Rogers also discovered similar identities for the modulus 7 :

$$
\begin{aligned}
& \prod_{\substack{n=1 \\
n \neq 0, \pm(\bmod 7)}}^{\infty}\left(1-q^{n}\right)^{-1}=\sum_{m=0}^{\infty} \frac{q^{2 m^{2}+2 m}}{\left(q^{2} ; q^{2}\right)_{m}}\left(-q^{2 m+2}\right)_{\infty} \\
& \prod_{\substack{n=1 \\
n \neq 0, \pm 2(\bmod 7)}}^{\infty}\left(1-q^{n}\right)^{-1}=\sum_{m=0}^{\infty} \frac{q^{2 m^{2}+2 m}}{\left(q^{2} ; q^{2}\right)_{m}}\left(-q^{2 m+1}\right)_{\infty} \\
& \prod_{\substack{n=1 \\
n \neq 0, \pm 3(\bmod 7)}}^{\infty}\left(1-q^{n}\right)^{-1}=\sum_{m=0}^{\infty} \frac{q^{2 m}}{\left(q^{2} ; q^{2}\right)_{m}}\left(-q^{2 m+1}\right)_{\infty} .
\end{aligned}
$$


Equations (4) and (6) first appeared in [4]. All three are proved by Rogers in [5]. A. Selberg rediscovered them in [6].

There is also a combinatorial theorem for the modulus seven. It is a special case of a combinatorial theorem by B. Gordon, [2], which was stated for all odd moduli greater than or equal to five.

(7) For $r=1,2$ or 3 , and any positive integer $n$, the partitions of $n$ into parts not congruent to $0, \pm r \bmod 7$ are equinumerous with the partitions of $n$ in which each part appears at most twice, the difference between nonidentical parts is at least two if either appears twice, and one appears as a part at most $r-1$ times.

While many proofs of statement (7) exist, until recently there was no proof which showed (7) as a direct consequence of equations (4)-(6). It was to supply such a proof that George Andrews introduced the notion of gap-frequency partitions (abbreviated g-f partitions) in [1]. The purpose of this paper is to provide a simpler derivation of the generating function for g-f partitions. This yields a more direct proof that equations (4)-(6) imply statement (7), and also leads to certain natural generalizations of $\mathrm{g}-\mathrm{f}$ partitions.

The generating function for g-f partitions.

Definition. A partition $\pi$ is said to be a gap-frequency (or g-f) partition if whenever a summand $s$ appears exactly $t$ times, the next larger part is at least $s+t$, and if it is exactly $s+t$ it can appear at most $t$ times.

ExAmPLe. $1+4+4+4+7+7+7$ is a g-f partition. Neither $1+3+3+3+6+6+6+6$ nor $2+3+5+5+5+7+7$ is a $g-f$ partition.

Definition. For positive integers $r, x$ and $n$, let $S_{r, x}(n)$ denote the number of g-f partitions of $n$ in which no part appears more than $x$ times and one appears at most $r-1$ times.

Theorem. For positive integers $r, x$ and $n$ and for $|q|<1$, let $M\left(m_{1}, \cdots, m_{x}\right)=M=\sum_{j=1}^{x} j^{2}\left(\begin{array}{c}m_{j} \\ 2\end{array}\right)+\sum_{1 \leqq i<j \leqq x} i j m_{i} m_{j}$. Then

$$
\sum_{m_{1}, \cdots, m_{x} \geqq 0} \frac{q^{M+m_{1}+2 m_{2}+\cdots+(r-1) m_{r-1}+2\left(r m_{r}+(r+1) m_{r+1}+\cdots+x m_{x}\right)}}{(q ; q)_{m_{1}}\left(q^{2} ; q^{2}\right)_{m_{2}} \cdots\left(q^{x} ; q^{x}\right)_{m_{x}}}=\sum_{n=0}^{\infty} S_{r, x}(n) q^{n} .
$$

For fixed values of $m_{2}, \cdots, m_{x}$, the left side of (8) can be summed using Euler's formula: 


$$
\sum_{m=0}^{\infty} \frac{q^{\left(\begin{array}{c}
m \\
2
\end{array}\right)} a^{m}}{(q ; q)_{m}}=(-a ; q)_{\infty}
$$

It is then a straightforward exercise to verify that when $x=2$ the right-hand sides of equations (4)-(6) are obtained.

Note that $S_{r, 2}(n)$ counts those partitions described in the second part of (7). The theorem is sufficient to prove that equations (4)(6) imply statement (7).

\section{Proof of the theorem.}

DEFINITION. A partition with attributes is a partition in which parts of equal value may be distinguished by some attribute or characteristic. For example, parts may be colored red, blue, green, etc. A partition with $x$ attributes is a partition in which at most $x$ attributes or characteristics are used. In a partition with $x$ attributes, each part will be denoted by an ordered pair, $\left(d_{i}, a_{i}\right)$, where $d_{i}$ is the value of the part and $a_{i}$ is its attribute, $1 \leqq a_{i} \leqq x$.

The generating function for partitions into exactly $m$ parts, each part greater than or equal to $b$ is given by

$$
q^{b m}(q ; q)_{m}^{-1}
$$

It follows that

$$
q^{b_{1} m_{1}+b_{2} m_{2}+\cdots+b_{x} m_{x}} x(q ; q)_{m_{1}}^{-1}(q ; q)_{m_{2}}^{-1} \cdots(q ; q)_{m_{x}^{-1}}^{-1}
$$

is the generating function for partitions with $x$ attributes such that for $1 \leqq i \leqq x$, there are exactly $m_{i}$ parts with attribute $i$, and each such part is greater than or equal to $b_{i}$.

DEFINITION. Let $R_{r}\left(m_{1}, \cdots, m_{x} ; n\right)$ denote the number of partitions of $n$ with $x$ attributes such that for $1 \leqq i \leqq x$ there are exactly $m_{i}$ parts with attribute $i$, each part with attribute $i$ is divisible by $i$, and all parts with attribute $i \geqq r$ are greater than or equal to $2 i$.

LEMma 1.

$$
\frac{q^{m_{1}+2 m_{2}+\cdots+(r-1) m_{r-1}+2\left(r m_{r}+(r+1) m_{r+1}+\cdots+x m_{x}\right)}}{(q ; q)_{m_{1}}\left(q^{2} ; q^{2}\right)_{m_{2}} \cdots\left(q^{x} ; q^{x}\right)_{m_{x}}}=\sum_{n=0}^{\infty} R_{r}\left(m_{1}, \cdots, m_{x} ; n\right) q^{n}
$$

Proof. This lemma follows from the discussion given above and the definition of $R_{r}\left(m_{1}, \cdots, m_{x} ; n\right)$.

DEFINITION. Let $S_{r}\left(m_{1}, \cdots, m_{x} ; n\right)$ denote the number of $\mathrm{g}-\mathrm{f}$ 
partitions of $n$ in which no part appears more than $x$ times, one appears at most $r-1$ times, and for $1 \leqq i \leqq x$, exactly $m_{i}$ different integers appear $i$ times.

LeMmA 2. Let $M\left(m_{1}, \cdots, m_{x}\right)=M=\sum_{j=1}^{x} j^{2}\left(\begin{array}{c}m_{j} \\ 2\end{array}\right)+\sum_{1 \leqq i<j \leqq x} i j m_{i} m_{j}$. Then $R_{r}\left(m_{1}, \cdots, m_{x} ; n-M\right)=S_{r}\left(m_{1}, \cdots, m_{x} ; n\right)$.

Before proving Lemma 2, we note that it and Lemma 1 imply the theorem, since

$$
\begin{aligned}
\sum_{n=0}^{\infty} S_{r, x}(n) q^{n} & =\sum_{m_{1}} \ldots m_{x} \sum_{x=0} S_{n=M} S_{r}\left(m_{1}, \cdots, m_{x} ; n\right) q^{n} \\
& =\sum_{m_{1}} \ldots m_{x} \geqq 0 \\
& \sum_{n=M}^{\infty} R_{r}\left(m_{1}, \cdots, m_{x} ; n-M\right) q^{n} \\
& =\sum_{m_{1}} \cdot \sum_{m_{x} \geqq 0} q^{M} \sum_{n=0}^{\infty} R_{r}\left(m_{1}, \cdots, m_{x} ; n\right) q^{n} \\
& =\sum_{m_{1}, \cdots, m_{x} \geqq 0} \frac{q^{M+m_{1}+\cdots+(r-1) m_{r-1}+2\left(r m_{r}+\cdots+x m_{x}\right)}}{(q ; q)_{m_{1}} \cdots(q ; q)_{m_{x}}}
\end{aligned}
$$

Proof of Lemma 2. We shall prove this lemma by establishing a one-to-one correspondence between partitions counted by $R_{r}\left(m_{1}, \ldots\right.$, $\left.m_{x} ; n-M\right)$ and those counted by $S_{r}\left(m_{1}, \cdots, m_{x} ; n\right)$.

Consider a partition counted by $R_{r}\left(m_{1}, \cdots, m_{x} ; n-M\right)$ with parts given by $\left(d_{1}, a_{1}\right),\left(d_{2}, a_{2}\right), \ldots$ and with the parts ordered from left to right such that if $d_{\lambda} / a_{\lambda}<d_{\mu} / a_{\mu}$, then $\left(d_{\lambda}, a_{\lambda}\right)$ precedes $\left(d_{\mu}, a_{\ell}\right)$ and if $d_{\lambda} / a_{\lambda}=d_{t^{\prime}} / a_{\mu^{\prime}}$ and $a_{\lambda}>a_{\ell !}$, then $\left(d_{\lambda}, a_{\lambda}\right)$ precedes $\left(d_{t \prime}, a_{\iota^{\prime}}\right)$. Clearly there is a unique such ordering of the ordered pairs.

This partition of $n-M$ with $x$ attributes is transformed into a partition of $n$ with $x$ attributes if each ordered pair $\left(d_{\lambda}, a_{\lambda}\right)$ is replaced by the pair $\left(e_{\lambda}, a_{\lambda}\right)$ where

$$
e_{\lambda}=d_{\lambda}+\sum_{k=1}^{\lambda-1} a_{\lambda} a_{k}
$$

We claim that our new partition is a partition of $n$. The total amount which has been added to our partition is

$$
\sum_{i=1}^{m_{1}+\cdots+m_{x}} \sum_{k=1}^{\lambda-1} a_{\lambda} a_{k}
$$

which is the second elementary symmetric function of the numbers

$$
\overbrace{1 \cdots 1}^{m_{1}} \overbrace{2 \cdots 2}^{m_{2}} \quad \ldots \quad \overbrace{x \cdots x}^{m_{x}},
$$

which is equal to 


$$
\sum_{j=1}^{x} j^{2}\left(\begin{array}{c}
m_{j} \\
2
\end{array}\right)+\sum_{1 \leqq i<j \leqq x} i j m_{i} m_{j}=M
$$

This proves our claim.

Observe that $a_{\lambda}$ divides $e_{\lambda}$, and for each $\lambda$

$$
\frac{e_{\lambda}}{a_{\lambda}}-\frac{e_{\lambda-1}}{a_{\lambda-1}}=\frac{d_{\lambda}}{a_{\lambda}}-\frac{d_{\lambda-1}}{a_{\lambda-1}}+a_{\lambda-1} \geqq a_{\lambda-1} \text {. }
$$

Equality occurs when $d_{\lambda} / a_{\lambda}=d_{\lambda-1} / a_{\lambda-1}$, which implies that $a_{\lambda-1} \geqq a_{\lambda}$. Also, if $a_{1} \geqq r$, then $e_{1} / a_{1}=d_{1} / a_{1} \geqq 2$. Thus if each part $\left(e_{\lambda}, a_{\lambda}\right)$ is replaced by $a_{\lambda}$ equal parts of value $e_{\lambda} / a_{\lambda}$, we have a partition counted by $S_{r}\left(m_{1}, \cdots, m_{x} ; n\right)$. This procedure is uniquely reversible (equal parts are added together and the resulting part is given the attribute equal to the number of parts which were added, $\sum_{\substack{i=1 \\ \hat{k}=1}} a_{\lambda} a_{k}$ is then subtracted from the value of the $\lambda^{\prime}$ th part), and so the one-toone correspondence is established.

This concludes the proof of Lemma 2, and so also the proof of the theorem.

\section{A generalization.}

DEFinition. A partition $\pi$ is said to be a $k$-fold $\mathrm{g}$-f partition if whenever a summand $s$ appears exactly $t$ times, then the next larger part is at least $s+k t$, and if it is exactly $s+k t$, it can appear at most $t$ times.

Definition. For positive integers $r, x, k$ and $n$, let $S_{r, x, k}(n)$ denote the number of $k$-fold $\mathrm{g}$-f partitions of $n$ in which no part appears more than $x$ times and one appears at most $r-1$ times.

By the method used above to find the generating function for $S_{r, x}(n)=S_{r, x, 1}(n)$, it can be readily verified that

$$
\sum_{n=0}^{\infty} S_{r, x, k}(n) q^{n}=\sum_{m_{1}, \cdots, m_{x} \geqq 0} \frac{q^{k 1+m_{1}+\cdots+(r-1) m_{r-1}+2\left(r m_{r}+\cdots+x m_{x}\right)}}{(q ; q)_{m_{1}} \cdots\left(q^{x} ; q^{x}\right)_{m_{x}}} .
$$

Since $S_{r, 1, k}(n)$ counts the number of partitions of $n$ into parts with minimal difference $k$, including at most $r-1$ ones, we see that the right sides of the Rogers-Ramanujan identities are special cases of the generating function for $S_{r, x, k}(n)$.

\section{REFERENCES}

1. G.E. Andrews, Gap-frequency partitions and he Rogers identities, (to appear).

2. B. Gordon, A combinatorial generalization of the Rogers-Ramanujan identities, Amer.

J. Math., 38 (1961), 393-399. 
3. G. H. Hardy and E. M. Wright, An Introduction to the Theory of Numbers, Fourth Edition, Oxford Univ. Press, London, 1960.

4. L. J. Rogers, Second memoir on the expansion of certain infinite products, Proc. London Math. Soc., 25 (1894), 318-343.

5. L. J. Rogers, On two theorems of combinatory analysis and some allied identities, Proc. London Math. Soc. (2), 16 (1917), 315-336.

6. A. Selberg, Über einige arithmetische Identitäten, Avhl. Norske Vid., 8 (1936).

Received July 31, 1979 and in revised form January 8, 1980. Partially supported by National Science Foundation Grant MCS 77-22992.

The Pennsylvania State University

UnIVERSity Park, PA 16802

Current address: University of Wisconsin

Madison, WI 53706 


\section{PACIFIC JOURNAL OF MATHEMATICS}

\section{EDITORS}

DONALD BABBITT (Managing Editor)

University of Galifornia

Los Angeles, California 90024

Hugo RossI

University of Utah

Salt Lake City, UT 84112

C. C. MoOre AND ANDrew OGG

University of California

Berkeley, CA 94720

\section{J. DugundjI}

Department of Mathematics University of Southern California

Los Angeles, California 90007

R. Finn and J. Milgram

Stanford University

Stanford, California 94305

ASSOCIATE EDITORS

E. F. Beckenbachi

B. H. NeumanN

F. WOLF

K. YOSHIDA

\section{SUPPORTING INSTITUTIONS}

UNIVERSITY OF BRITISH COLUMBIA

CALIFORNIA INSTITUTE OF TECHNOLOGY

UNIVERSITY OF CALIFORNIA

MONTANA STATE UNIVERSITY

UNIVERSITY OF NEVADA, RENO

NEW MEXICO STATE UNIVERSITY

OREGON STATE UNIVERSITY

UNIVERSITY OF OREGON
UNIVERSITY OF SOUTHERN CALIFONIA

STANFORD UNIVERSITY

UNIVERSITY OF HAWAII

UNIVERSITY OF TOKYO

UNIVERSITY OF UTAH

WASHINGTON STATE UNIVERSITY

UNIVERSITY OF WASHINGTON

Printed in Japan by International Academic Printing Co., Ltd., Tokyo, Japan 


\section{Pacific Journal of Mathematics \\ Vol. 89 , No. 1 \\ May, 1980}

David Bressoud, A note on gap-frequency partitions ................ 1

John David Brillhart, A double inversion formula ................. 7

Frank Richard Deutsch, Günther Nürnberger and Ivan Singer, Weak

Chebyshev subspaces and alternation .......................

Edward Richard Fadell, The relationship between Ljusternik-Schnirelman

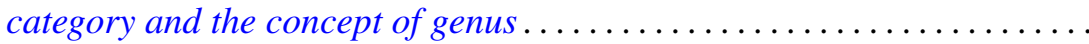

Harriet Jane Fell, On the zeros of convex combinations of polynomials.......

John Albert Fridy, An addendum to: "Tauberian theorems via block

dominated matrices" ..................................

Andrzej Granas, Ronald Bernard Guenther and John Walter Lee, Applications of topological transversality to differential equations. I. Some nonlinear

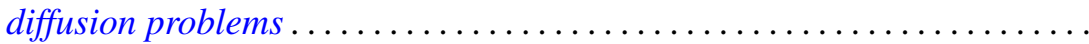

David E. Handelman and G. Renault, Actions of finite groups on self-injective

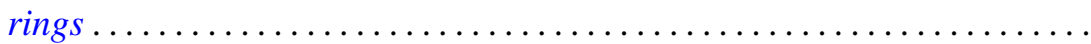

Michael Frank Hutchinson, Local $\Lambda$ sets for profinite groups .............

Arnold Samuel Kas, On the handlebody decomposition associated to a

Lefschetz fibration...

Hans Keller, On the lattice of all closed subspaces of a Hermitian space.....

P. S. Kenderov, Dense strong continuity of pointwise continuous

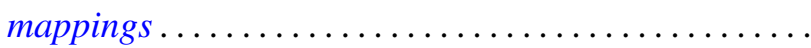

Robert Edward Kennedy, Krull rings.................

Jean Ann Larson, Richard Joseph Laver and George Frank McNulty,

Square-free and cube-free colorings of the ordinals ...

Viktor Losert and Harald Rindler, Cyclic vectors for $L^{p}(G)$

John Rowlay Martin and Edward D. Tymchatyn, Fixed point sets of

1-dimensional Peano continua...

Augusto Nobile, On equisingular families of isolated singularities ...

Kenneth Joseph Prevot, Imbedding smooth involutions in trivial bundles ...

Thomas Munro Price, Spanning surfaces for projective planes in four space.

Dave Riffelmacher, Sweedler's two-cocycles and Hochschild cohomology....

Niels Schwartz, Archimedean lattice-ordered fields that are algebraic over

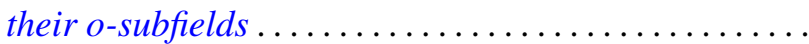

Chao-Liang Shen, A note on the automorphism groups of simple dimension groups.

Kenneth Barry Stolarsky, Mapping properties, growth, and uniqueness of

Vieta (infinite cosine) products ...

Warren James Wong, Maps on simple algebras preserving zero products. I.

The associative case ............................. 